\title{
COMUNICACIÓN Y EDUCACIÓN Un balance cualitativo
}

\author{
María Dolores Cáceres Zapatero
}

Profesora de Teoría de la Comunicación. Universidad Complutense de Madrid.

\section{Resumen}

Este trabajo es una revisión bibliográfica de la producción científica llevada a cabo en España a propósito de la relación entre medios de comunicación y educación. Se ha considerado la investigación realizada por autores españoles y publicada en España durante la última década (19962006). Se abordan los trabajos que tratan los medios de comunicación en general y, en particular, la televisión y los nuevos medios. La tema de la educación se plantea con especial atención a los sectores infantil y juvenil.

\section{Palabras clave}

Medios de Comunicación - Medios Audiovisuales - Televisión - Nuevas Tecnologías - Educación Infancia

\begin{abstract}
This paper is a bibliographical revision of the scientific production that has been carried out in Spain about the relation between the media and education. The investigation done by spanish authors and published in Spain during the last decade (1996-2006) has been considered. The projects that treat the media in general and, particularly, the television and new media are tackled. The education theme is treated with special care for the children and teenagers' sectors.
\end{abstract}




\section{Key words}

Media of communication - Audiovisual Media -Television - New Technologies - Education Children

\section{Introducción}

El objeto de este trabajo es hacer una revisión de la producción bibliográfica llevada a cabo en España en el último decenio (desde el año 1996 hasta 2006, ambos inclusive) sobre medios de comunicación y educación.

Cómo se trataba de hacer el estado de la cuestión en nuestro país, la revisión se ha ceñido a los trabajos llevados a cabo por autores españoles, relativos al contexto de la educación y los medios en nuestro país y publicados en España, tanto en revistas de carácter científico como libros. No se han tenido en cuenta las tesis doctorales inéditas, puesto que la intención de esta colaboración es hacer el estado de la cuestión de la investigación publicada.

La temática que aborda este trabajo es la relación entre medios de comunicación y educación. Esta relación se plantea desde las distintas y amplias perspectivas que cabe considerarla. Por una parte se han tomado los medios de comunicación en general y, en particular los audiovisuales y nuevos medios, y por otra parte, la educación con especial atención a la infancia y a los jóvenes que, por estar en período de formación, se muestran especialmente sensibles la presión de los medios.

La mirada a esta temática está dirigida desde los medios hacia la educación y no viceversa, aunque ambos términos tengan igual relevancia. Esta matización ha sido importante a la hora de plantear la búsqueda bibliográfica que obligaba a introducir un orden en los términos de la misma y resulta relevante a la vista de los resultados obtenidos: aunque el planteamiento inicial es ir de los procesos comunicación hacia la educación, son los educadores e investigadores en este campo los que muestran mayor interés por estudiar los medios de comunicación.

Las relaciones entre ambos tópicos son evidentes: todo proceso educativo se sustenta sobre interacciones comunicativas diversas. Interesaba en este momento, hacer una puesta al día de cuáles son las tendencias y los tópicos recurrentes relativos al ámbito de la educación que se plantean desde los medios de comunicación. Dicho en otros términos, cuáles son los problemas fundamentales que abordan los investigadores españoles, cuáles son las virtualidades de la tecnología comunicativa y cuál es su aprovechamiento en el ámbito educacional, cuáles son los retos y las asignaturas 
pendientes en el ámbito escolar, qué propuestas de futuro, innovadoras o conservadoras, se presentan para el sistema educativo a la luz de los nuevos medios.

En conjunto se han examinado más de tres centenares de referencias extraídas de las principales bases de datos bibliográficas y un buscador especializado (Google Scholar).

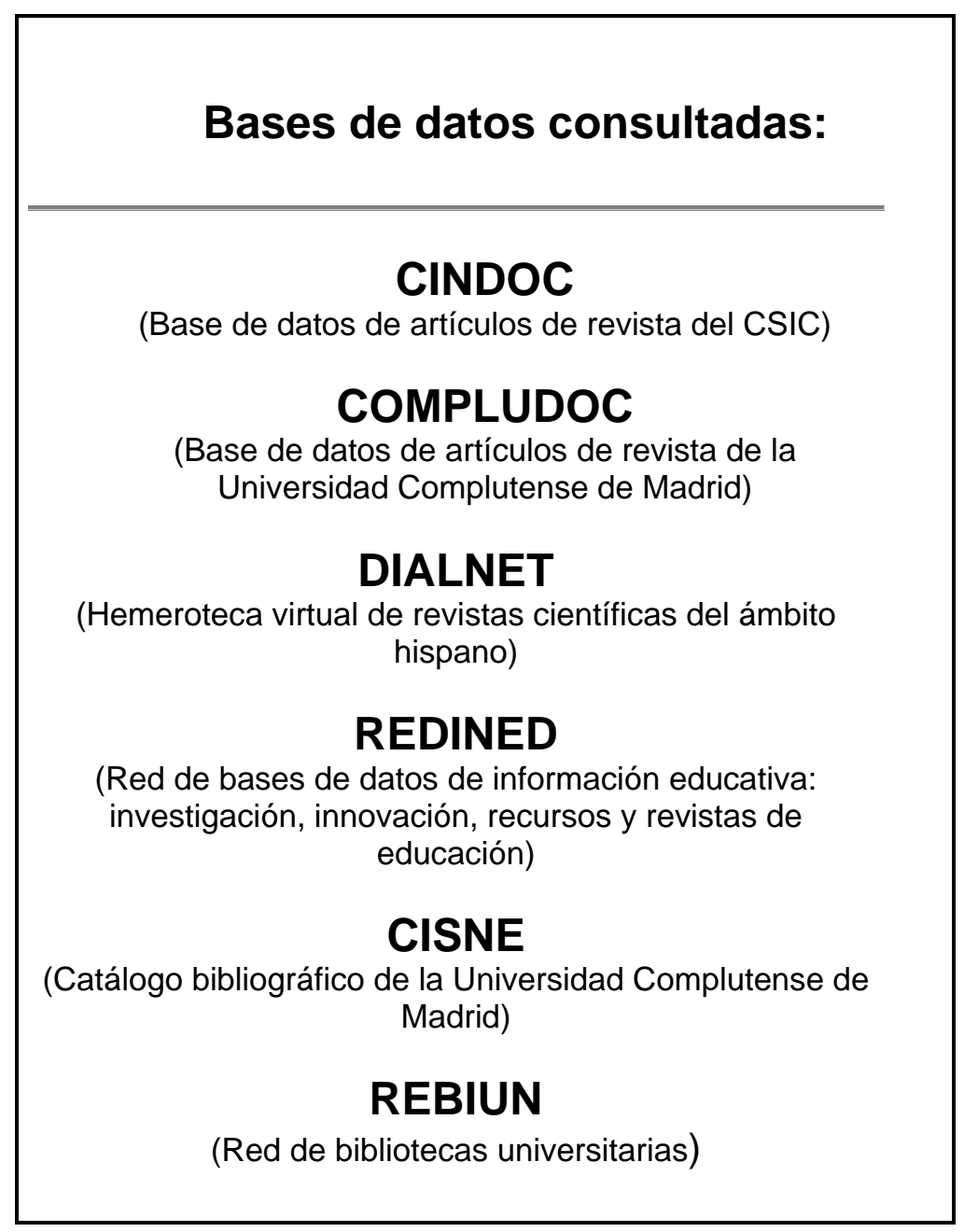

\section{Tendencias}

En la última década hay una voluminosa producción que se ocupa de abordar el estudio de la relación entre medios de comunicación y educación. Cuando se examina la producción intelectual en las publicaciones periódicas, sobresale el hecho de que en este binomio parece llevarse a cabo más investigación desde el ámbito de la educación que desde los propios medios. Dicho en términos 
diferentes, más de la mitad de las publicaciones son revistas que pertenecen ámbito de la educación ${ }^{1}$. El examen de las cuarenta y cuatro revistas consideradas, revela que existe una mayor preocupación en los investigadores del área de la educación por el estudio de los medios, sus potencialidades y sus aplicaciones, que por parte de los estudiosos de los medios por considerar la dimensión educativa de los mismos. Hay honrosas excepciones entre las revistas dedicadas al estudio de la comunicación como por ejemplo: Telos (Revista de pensamiento sobre tecnología y sociedad) que periódicamente se ocupa del tema de los usos educativos de los medios y, en los últimos años, de las tecnologías de información y comunicación en los nuevos entornos de aprendizaje. Entre las revistas del área de las Ciencias Sociales, llama la atención la poca presencia de los tópicos estudiados. En los últimos años, la aparición de revistas digitales ha dado cabida a trabajos que estudian la comunicación y la educación, sobre todo con relación a los medios audiovisuales y a las nuevas tecnologías.

La producción bibliográfica es abundante en lo que se refiere sobre todo a la televisión y, en los años recientes, a la tecnología educativa.

\section{Los tópicos de investigación}

Existe una producción abundante dedicada a medios de comunicación con relación al tema de la educación. Esta voluminosa producción, se reparte entre medios audiovisuales -con clara preponderancia de la televisión- y tecnologías de la información y comunicación. La distribución cronológica de estos tópicos reproduce el desarrollo que han seguido esos mismos medios en la sociedad. En los primeros años del decenio considerado, hay un decisivo protagonismo de la televisión que luego, en años más recientes, se verá acompañada por los nuevos medios. Son escasos sin embargo los trabajos que se ocupan de los medios impresos o la radio.

Si bien hay que apuntar la importancia que tienen en el momento presente tópicos tan recurrentes como la publicidad en televisión, Internet o los videojuegos, en esta revisión bibliográfica, no se incluye un análisis monográfico de ellos toda vez que, por su entidad, merecerían sendos trabajos específicos imposibles de abarcar en este intento.

Cabe destacar la escasez de trabajos de carácter metodológico. Apenas se ha encontrado alguna referencia relativa a la complejidad de la audiencia infantil y a los problemas que resultan para su medición en el aula, apuntando alguna propuesta concreta en este sentido.

\footnotetext{
${ }^{1}$ Ver ANEXO 1: listado de revistas
} 
La problemática de los medios de comunicación con relación al tema de la educación se aborda tanto, a nivel general, señalando su importancia como medios de transmisión de cultura y aprendizaje social, como a nivel particular, dando lugar a estudios sectoriales que se centran en un solo medio, en general la televisión, en algún caso con relación a la prensa o la radio y, en los últimos años, las nuevas tecnologías y el multimedia.

La influencia socializadora y formadora de los medios es planteada en la literatura científica con relación a temas diversos de gran trascendencia: adquisición de estereotipos sexuales, relaciones interpersonales, educación ciudadana, preparación para la vida cotidiana, educación en valores; u otros que se relacionan con la sensibilidad social presente en cada momento, por ejemplo la multiculturalidad, educación para paz, transmisión de valores para prevenir la violencia en la escuela y de género, generar una cultura de convivencia democrática, educación para el desarrollo, y todo un repertorio amplio de tópicos que se asocian con los conflictos y problemas actuales que pueden darse en cualquier parte del mundo y que están presentes en la vida del alumnado a través de los medios de comunicación. Sin olvidar, señalan algunos autores, las contradicciones que, en ocasiones, se generan a partir de postulados opuestos: los presentados por parte de la escuela y los transmitidos en educación informal.

La relación entre medios de comunicación y educación es abordada teniendo en cuenta las especiales características de la infancia y los jóvenes que, por estar en período de formación, se muestran especialmente sensibles al impacto de los medios. Hay trabajos que abordan específicamente esta problemática desde el ámbito estrictamente infantil, teniendo en cuenta sus peculiaridades frente a otros grupos de edad, y otros, con un enfoque más amplio, que incluyen otros segmentos de edad desde las características que les son comunes.

Aunque el medio hegemónico es sin lugar a dudas la televisión, hay publicaciones que también toman en consideración los otros medios intentando hacer un aprovechamiento de las capacidades de cada uno de ellos, habida cuenta de que conforman gran parte de los conocimientos previos con los que el alumnado llega al aula y, por consiguiente, pueden ser ventajosamente aprovechados desde el punto de vista didáctico. Por ejemplo, propuestas de carácter teórico-práctico: organización de talleres de prensa para conseguir aprendizajes significativos a través del trabajo en grupo y fomentar actitudes colaborativas; explorar las posibilidades de la radio educativa llevando a a cabo proyectos de radio escolar (creación de una emisora escolar para fomentar entre los alumnos la reflexión sobre los medios), o la realización por jóvenes de un Instituto de Enseñanza Secundaria de un programa de televisión dirigido también a jóvenes. $\mathrm{O}$, en otro orden de cosas, se propone el 
análisis de los medios, por ejemplo la prensa escrita, para comprender mejor las imágenes y opiniones que la población se ha formado a propósito de la educación.

En la producción bibliográfica estudiada, se reconoce de manera unánime la necesidad de introducir los medios en la escuela para aprovechar sus potencialidades, tanto en lo que se refiere a capacidades técnicas (por ejemplo la utilización del vídeo o el retroproyector dentro del aula, los medios audiovisuales en relación a la educación artística) como para desarrollar otras capacidades relacionadas directamente con el currículum escolar, por ejemplo: el análisis de los medios para aprender a argumentar y desvelar el verdadero sentido e intención de un mensaje. Especial importancia tiene el aprovechamiento que cabe hacer de los medios de cara a las alumnas y los alumnos con necesidades de educación especial (por ejemplo, rehabilitación del lenguaje en alumnos con deficiencias) o en las escuelas de sectores populares.

Los medios tienen una gran influencia en la construcción del pensamiento social en especial en niños y jóvenes. Ante esto la educación formal no puede permanecer pasiva, por consiguiente se impone llevar a cabo una auténtica alfabetización en medios que forme receptores críticos y preconice un consumo racional de los mismos. Se trata de que las alumnas y alumnos sean capaces de informarse, comprender y asimilar la información, contrastar intereses y puntos de vista, interpretar lo que está pasando y proponer soluciones a partir del diálogo y el respeto mutuo. Dentro de una didáctica crítica, se señala la importancia de estudiar los medios para discernir entre ficción y realidad como medio que pueda conducir a una movilización y participación social conscientes. En última instancia, una adecuada alfabetización audiovisual contribuiría a resolver la tensión entre las influencias supuestamente negativas y aquellas que les atribuyen un papel democratizador por su capacidad de reconocer y dar validez en el currículo escolar, a las culturas extraescolares de las alumnas y alumnos.

En el abordaje de los medios de comunicación, y en especial de los medios audiovisuales, con relación a la educación, no faltan desde luego los trabajos que, con un enfoque psicológico, por ejemplo, analizan los fenómenos emocionales con relación a las cuestiones de percepción de la imagen, aquellos que se plantean en términos comparativos con otros países de nuestro entorno cercano (Francia, Italia, Reino Unido), o los que se sitúan a un nivel macrosocial. Se consideran las complejas relaciones entre los aspectos comerciales de la cultura de masas audiovisual y sus potencialidades educativas o empobrecedoras; otros desde la psicología del desarrollo infantil y juvenil reflexionan sobre el contexto audiovisual como una fuente importante en la construcción del conocimiento individual y social de niños y jóvenes. No faltan las valoraciones del binomio creación-cultura frente a negocio-alienación, sugiriendo la necesidad de un protagonismo activo por 
parte de padres y educadores. Destacan los trabajos que enfatizan la labor importantísima de transmisión de valores en general y en particular, en estudios sectoriales dedicados a ámbitos concretos, como la formación política, la violencia, las diferencias de género, a visión del cuerpo en nuestra sociedad, el deporte, etc.

Otra vertiente de la investigación publicada es la que aborda los medios con relación a profesores y educadores. Por una parte la introducción de los medios en la escuela plantea la cuestión de la predisposición de los profesores hacia los medios y, por otra, el grado de conocimiento que es necesario para un adecuado aprovechamientos de todas sus posibilidades dentro del aula. A estos efectos, se han elaborado y publicado distintas guías de medios audiovisuales o cuadernos de trabajo para el alumnado. La cuestión de la formación y la responsabilidad de los profesionales de la educación en el uso de los medios, tomará de nuevo vigencia en los últimos años, con la introducción de las nuevas tecnologías de la información y la comunicación.

\subsection{La televisión: el medio hegemónico}

Dentro de toda la producción intelectual dedicada a los medios audiovisuales destaca sobre todos los demás tópicos, la televisión como medio hegemónico y omnipresente en la cotidianidad de niños y jóvenes, de ahí la necesidad compartida de acercarse al conocimiento del medio.

Las cuestiones planteadas en torno al poder y alcance de los medios en las últimas décadas sobre todas las capas de la sociedad al margen de la edad, sexo, extracción social o nivel cultural, se traslada, casi en los mismos términos, al ámbito de la televisión. Así encontramos tópicos y planteamientos que reiteran lo expuesto.

El reconocimiento de que la televisión es el principal medio de transmisión de cultura para amplias capas de la población, agente importante de conformación del imaginario social y de las formas de la socialidad, está presente en numerosos trabajos que plantean la necesidad de estudiar el medio con relación a su potencial educador.

El binomio televisión-educación ha atravesado históricamente distintas etapas desde la aparición del medio en España. Desde sus orígenes pasada la mitad de los años cincuenta, hasta la transición política, puede hablarse de desencuentro entre ambas, sin que se produzca un aprovechamiento del medio en el ámbito de la educación. Durante la etapa democrática, el nuevo contexto constitucional recién estrenado, hace que exista una notable preocupación por ejemplo, por la calidad de la información y la salvaguarda de su carácter democrático; pero la programación 
educativa no parece correr la misma suerte. Salvo honrosas excepciones, no se producen cambios sustanciales que permitan hablar de una auténtica televisión educativa en España (Pérez Tornero, 2000). En los años recientes, la preocupación de los gobiernos respecto a la televisión ha girado en torno a la definición del modelo de televisión pública en todas sus dimensiones: institucionales, económicas, de funcionamiento, etc. En el momento actual, la televisión atraviesa una etapa de cambios y redefinición a raíz de los compromisos electorales contraídos por el partido ganador de las últimas elecciones legislativas celebradas en 2004, pero hasta el momento presente no parece que los cambios pasen de hecho, por un compromiso serio con la educación. Tampoco puede decirse que en las televisiones autonómicas haya habido una apuesta decidida por una programación cultural y educativa.

El modelo audiovisual español se caracteriza por una televisión de mercado, de competencia muy dura entre cadenas que, desde una oferta múltiple y similar, luchan por la audiencia y se disputan el mercado de la publicidad. En este contexto, se producen continuos recortes de programas de índole cultural a favor de otros más rentables que alejan a la televisión de la vocación de servicio público presente en su propia definición.

Por otra parte en la producción académica sobre el tema, existe una conciencia clara de que la influencia atribuible a la televisión obedece de forma importante a la acción continuada del medio, su ubicuidad y la duración de la exposición; dicho en otros términos los efectos atribuibles a los medios son a largo plazo resultado de un proceso acumulativo constante. Así se detecta una preocupación por las altas cuotas de consumo televisivo entre el público infantil. En el momento actual, el tiempo medio de permanencia frente al televisor se cifra, para la población en general, en torno a las tres horas y media diarias aproximadamente, permaneciendo esta cifra más o menos constante en la última década. Para la franja de edad que comprende los niños de 4 a 12 años, el consumo televisivo se sitúa en la última temporada en 154 minutos (Geca, 2005) habiendo aumentado respecto a la temporada anterior. Sólo se produce un descenso en la exposición a medio en los jóvenes de 13 a 24 años, debido sin duda a la migración hacia los nuevos medios, principalmente Internet.

En la exposición al medio también se aprecian cambios notables: se pasado de un consumo grupal a un consumo atomizado. La imagen tradicional de toda la familia reunida viendo la televisión ha pasado a la historia y la tendencia es, cada vez más rotunda, hacia un consumo individualizado. El aumento del número de aparatos receptores por hogar, junto con las transformaciones técnicas operadas que permiten una personalización en cuanto a la elección y visionado del programa deseado -televisión a al carta-, y la diversidad de la oferta de contenidos, ha 
producido una atomización de la audiencia y nuevas pautas en la forma de ver la televisión. Ha aumentado significativamente el número de telespectadores que ven a solas la televisión una gran parte del tiempo de su exposición a la televisión, lo que conduce a pensar que la fruición del medio ya no consiste en compartir su exposición con los demás. Más bien induce a pensar que es una vía de reforzamiento del individualismo característico de nuestra sociedad, aunque esta tendencia social disgregadora convive con otra convergente, como por ejemplo las nuevas formas de comunicación generadas a partir de la implantación y el uso extendido de Internet -sobre todo entre los jóvenes-, sin olvidar un hecho importante: dichos procesos carecen de interacción directa.

La aparición de la televisión en el campo de la educación como tópico de investigación presenta en general un carácter crítico y reivindicativo, tanto en lo que se refiere al análisis de los contenidos, como en lo relacionado con la necesidad de formar receptores críticos y de inculcar hábitos racionales de consumo. Cabe destacar el apartado dedicado al estudio de los efectos de la televisión en el desarrollo infantil y cómo la exposición al medio afecta evolutivamente a las niñas y niños en las distintas etapas que configuran las grandes funciones psicológicas como el desarrollo cognitivo, la atención, la imaginación, la construcción de la realidad, la identidad, etc.

Respecto a la programación de la televisión, son objeto de estudio tanto los contenidos de carácter estrictamente educativo como otros que tienen un potencial socializador. También se han considerado también los rasgos y características de la programación y producción orientadas a la cultura y su divulgación, e incluso el propio estilo comunicativo de la televisión educativa. Existen trabajos que abordan el análisis de emisiones concretas (por ejemplo "La bola de cristal”, "La aventura del saber”) o que hacen un recorrido por los mejores programas de contenido educativo.

Desde una perspectiva crítica, se cuestiona la calidad de los contenidos de la televisión, reclamando en consecuencia, un necesario control sobre la programación dirigida específicamente a la infancia por la especial trascendencia que tiene en este segmento de la audiencia especialmente vulnerable por estar en período de formación.

Habida cuenta de este hecho, se plantea la necesidad de un debate sobre la calidad de esta programación, el establecimiento de parámetros desde los cuales definir una deontología profesional y reivindicar una actuación ética entre todos aquellos que intervienen en el tratamiento de la programación infantil y juvenil, así como una legislación de protección de los menores frente a la televisión. Destacan en este sentido los trabajos dirigidos al examen y necesidad de la aplicación severa de códigos de autocontrol. Este tema vigente para la televisión se hará, posteriormente, extensivo a los nuevos medios tecnológicos. 
Toda vez que la televisión modela y configura en todos los órdenes la vida de los ciudadanos, la escuela se plantea como una necesidad reflexionar sobre el medio y sobre el inevitable maridaje televisión-educación. Esta preocupación se traduce en distintas líneas de trabajo. En primer lugar, aquella que incide en la formación de competencias para que niños y jóvenes se conviertan en receptores críticos. Se trataría de proporcionales el suficiente conocimiento del medio (comprender su lenguaje, entender su discurso, conocer su tecnología) para que se apropien de la televisión en su propio beneficio. Esta necesaria alfabetización televisiva contribuiría a impermeabilizar a este segmento de la audiencia frente a efectos indeseables, como de la presencia de contenidos violentos, inducción desmesurada al consumo por parte de la publicidad, hábitos insanos de vida, etc. En este sentido, hay un buen número de trabajos que se ocupan sectorialmente de los efectos del medio en distintos ámbitos: educación sexual, ámbito sanitario, rendimiento deportivo, conformación de la fantasía a partir de programas de dibujos animados, representación de conductas violentas, percepción del terrorismo, etc

En resumen, en palabras del profesor Aguaded “La comprensión intelectual del medio, la lectura crítica y desmitificación de sus mensajes, así como la capacidad para su utilización libre y creativa, desde un saber pragmático, se nos revela como la clave para la educación de la competencia televisiva” (Aguaded, 1999, p.17).

En esta línea de trabajo también hay que destacar el papel que juegan en la formación de niños y jóvenes como espectadores, otros entornos interaccionales que tienen responsabilidad en el desarrollo de su relación con el medio: los contextos familiares, profesionales, cívicos y políticos. Especial atención merece en la producción bibliográfica, el tópico familia-televisión. El consumo televisivo se produce principalmente en el hogar. Si bien es cierto, como ya se ha apuntado, que la tendencia son las pautas individualizadas de consumo debido a la multiplicación de aparatos receptores de televisión en el hogar, son, no obstante, los padres y la familia uno de los escenarios básicos para entender el contexto de la recepción junto con el grupo de iguales -sobre todo entre adolescentes- que actúa como filtro de interpretación, incitador de consumo y adquisición de modelos de referencia.

La segunda de las líneas de trabajo apuntadas explora las posibilidades culturales y educativas de los contenidos de la televisión, aunque a veces dichas potencialidades hayan quedado ensombrecidas por el prejuicio de los profesores o el poco interés de los profesionales del medio que a menudo descuidan el lugar que la programación educativa debe ocupar en el servicio público. En este sentido, se reclama la necesidad de una televisión educativa y se estudian algunas de las experiencias más significativas. 
Una tercera línea de investigación reclama constantemente desde la escuela, el maridaje entre televisión y educación, ya sea formando parte la primera del currículo escolar o como instrumento al servicio del aprendizaje. En efecto, desde la reforma educativa ha existido un intento de adaptación al entorno que ha dado lugar a una mayor flexibilización del currículo escolar, haciéndolo menos lineal y más modular y también menos estricto y más abierto y, por consiguiente, más permeable a otras formas de aprendizaje. Sin necesidad de constituir una disciplina concreta, la televisión debe formar parte de diferentes materias de manera transversal; por ejemplo en asignaturas como lengua, arte, tecnología, historia, ciencias sociales. El análisis de la construcción narrativa de ciertos contenidos, el examen de las estructuras profundas del mensaje y los valores presentes en algunos programas pueden favorecer el desarrollo de la conciencia crítica de los alumnos y su comprensión axiológica de la vida.

Como se ha dicho anteriormente, no es objeto de este trabajo considerar monográficamente un tema muy directamente relacionado con la televisión como es la publicidad. No obstante, hacer una breve referencia es insoslayable, puesto que son numerosos los trabajos que abordan el tema colateralmente. Se plantean cuestiones importantes como la influencia inconsciente de los anuncios, la violencia como recurso creativo o el potencial educativo de la publicidad, por ejemplo para potenciar determinados hábitos saludables en la infancia (verbigracia, respecto a hábitos alimentarios, práctica deportiva, etc.) que, de por sí, merecerían un trabajo monográfico. En este sentido y desde una perspectiva crítica de los medios, se encuentran análisis comparativos de la visión del ser humano que presenta la publicidad respecto a cuestiones concretas como la amistad, la familia, la belleza, etc. con la vida real.

\subsection{La educación en el nuevo milenio}

Las nuevas tecnologías son un fenómeno de amplio espectro cuyas consecuencias se dejan sentir en todos los ámbitos de la vida social y también en el de la educación, como no podía ser menos. Así, en los últimos años, aparece como tópico importante la tecnología educativa, a la sombra del auge cobrado por las nuevas tecnologías de información y comunicación. La voluminosa producción en esta línea, es un buen indicador de la preocupación de la sociedad en general y de los colectivos profesionales especializados en particular por los medios tecnológicos y su virtualidad educativa. Buena prueba son los congresos, investigaciones, jornadas, encuentros y foros de debate celebrados 
en España bajo la rúbrica de comunicación, tecnología y educación (Pérez, 2002). Son numerosos los textos -más de un tercio de las referencias analizadas- que, en los últimos años, se ocupan del tópico tecnología educativa, abordándola desde sus diversas definiciones, desde los fundamentos teórico-prácticos que permiten orientar la producción y el uso de los medios tecnológicos instruccionales de manera efectiva o desde una visión histórica que permite entenderla como una disciplina que ha evolucionado en pos de responder al contexto educativo por donde ha transitado en cada momento. Hay trabajos que recogen el estado actual del tema y otros que hacen un esfuerzo de prospectiva.

Las nuevas formas de concebir la comunicación y el conocimiento en una sociedad marcada por el auge y desarrollo de la información y las telecomunicaciones, y la incorporación de éstas al contexto educativo como una vía de innovación, dirigen la mirada a explorar sus posibilidades y sus limitaciones, así como sus implicaciones sociales aunque, se señala también, no todo en ellas es nuevo. El potencial innovador de las tecnologías aplicadas a los proyectos educativos, pasa necesariamente por la manera de concebir dichos proyectos y llevarlos a la práctica.

Las tecnologías del conocimiento afectan al pensar y al sentir, lo cual ha creado un nuevo entorno comunicacional que modifica nuestra manera de percibir y vivir en el mundo -y también de aprender-, no exento de valoraciones: la tecnología no es neutra. Las tecnologías digitales abren y amplían el campo de la información y el entretenimiento, pero también

...más allá de un conjunto de medios más o menos sofisticados, las nuevas tecnologías de la información deben ser valoradas como un nuevo paradigma con un importante impacto cultural. Y aquí, el término paradigma es utilizado en su significación más vinculada a la idea de cambio irreversible o revolución conceptual y metodológica. (De Pablos y Jiménez, 1998, p. 9).

Habida cuenta de esta revolución tecnológica que propicia una trasformación en los procesos de aprendizaje, se pone de manifiesto la necesidad de una mirada crítica que ponga la tecnología al servicio de los seres humanos y ofrezca igualdad de oportunidades al mayor número de ciudadanos.

La producción del conocimiento y la distribución del saber han desbordado las instituciones formales de educación. Por consiguiente, se hace indispensable pensar la educación más allá de las fronteras del aula y de las paredes del colegio. Desde aquí se plantea pensar en los nuevos medios y en sus efectos, aprovechar sus virtualidades y considerar sus limitaciones, sin olvidar la frustración 
por no haber alcanzado las expectativas iniciales. Por ejemplo no siempre se ha producido una integración de Internet en la escuela a la velocidad que cabría esperar. Ante esta situación se impone analizar las causas y proponer líneas de actuación para estructurar el trabajo en el aula partiendo de la situación real en el momento presente.

La nueva sociedad de la información requiere que las instituciones educativas se abran a la diversidad de una realidad heterogénea y cambiante. Se plantea una reconceptualización de la noción de educación: ir a una visión más amplia y flexible en todas sus dimensiones, abordar el papel de las propias instituciones educativas y arrogarse con responsabilidad la explotación plural e innovadora que puede hacerse de los nuevos medios.

Los nuevos medios son un instrumento de gran potencialidad y ayuda en al campo de la educación. Conocer las posibilidades de las tecnologías de información y comunicación puede ayudar a trasformar los espacios de formación además de ser un buen recurso para los propios educadores. Las nuevas formas de comunicación que posibilitan las tecnologías digitales (foros, chats, blogs, correo, etc.) conllevan importantes cambios metodológicos en las estrategias didácticas y la creación de entornos virtuales de aprendizaje. Lograr que estos cambios sean de calidad implica transformaciones en la concepción de los alumnos-usuarios, en los profesores y en la Administración con relación al diseño de la enseñanza y de los sistemas de comunicación que la institución establece.

Las nuevas tecnologías rompen las barreras entre conocimiento escolar y extraescolar, se señala. En este sentido, deberían servir para resolver las situaciones que se producen en la sociedad actual, ligadas a procesos formativos no presenciales: formación continua, a distancia, flexible y adaptada; y también al servicio de personas con necesidades especiales, formación de adultos, educación popular o colectivos desfavorecidos. Por otra parte, las distintas posibilidades educativas que permite Internet deberían generar un nuevo alumnado centrado en la búsqueda, clasificación y elaboración de la información. Del mismo modo que se están produciendo cambios en la mentalidad y en la cultura docente, dichos cambios deberían también alcanzar a los estudiantes que pueden responder con flexibilidad y autonomía a sus necesidades individuales de formación, ritmo de aprendizaje, disponibilidad horaria, etc., y favorecer la comunicación multidireccional y el trabajo colaborativo.

Un tema recurrente en la investigación publicada es el reto que se le plantea a la escuela en la sociedad de la información. ¿Cómo puede y debe la institución educativa hacer frente a las nuevas necesidades del alumnado, a las nuevas prácticas educativas que se generan y a la necesaria formación y reciclaje de los docentes en el ámbito tecnológico? 
Asumir la sociedad de la información supone, desde el punto de vista de la educación por una parte, incorporar estas tecnologías como objetivo mismo de la educación. Es decir, acercar a los alumnos y alumnas al conocimiento de los nuevos medios, a fin de conseguir la suficiente alfabetización y combatir un factor de desigualdad de acceso y exclusión social. Se propone el concepto de alfabetizaciones digitales para los cambios que suponen las nuevas prácticas multimediales (Rodríguez Illera, 2004) y escapar a una concepción funcional, basada en el simple aprendizaje de determinadas técnicas -y alguna competencia asociada-: aprender a usar los ordenadores, las aplicaciones generales, el procesador de textos, el correo electrónico, los navegadores, etc. Claro está que dichas destrezas son necesarias, pero concebirlas como únicas, reduce la alfabetización digital a un conjunto de aplicaciones.

Esta sensibilidad se ha traducido, en primer lugar, en la introducción de materias concretas de diversa denominación en el currículo académico de las enseñanzas. Y, en segundo lugar, en una perspectiva transversal, que coloca los recursos técnicos (archivos digitales históricos, aplicaciones musicales en CD-Rom) al servicio del proceso de aprendizaje, en distintas asignaturas, como por ejemplo las disciplinas artísticas, las Ciencias Sociales, el dibujo, el aprendizaje de lenguas, la Historia, etc.

\section{Competencias profesionales del docente en el siglo XXI}

En este contexto, las competencias docentes y el desarrollo profesional de los educadores se encuentra obviamente sometido al influjo de la sociedad de la información y la comunicación. Todo ello ha conducido a un replanteamiento de las competencias profesionales del profesor en el ámbito tecnológico, produciendo cambios en su mentalidad respecto a las exigencias que debe afrontar de cara a un buen desarrollo competencial. Se plantea el grado de predisposición y preparación de los futuros profesores para el uso de aparatos y dispositivos tecnológicos asociados a los procesamientos digitales de información, así como la imagen que los profesores tienen de la tecnología y el papel de la Administración para facilitar los recursos necesarios a los efectos de mejorar dicha capacitación.

En este sentido hay trabajos que plantean reflexiones más o menos teóricas sobre la calidad de la educación ante los retos que presenta la sociedad de la información: qué líneas de mejora cabe plantearse desde las vertientes instructiva, institucional, curricular y cultural, ni otros de carácter más práctico como, por ejemplo, la elaboración de guías multimedia para docentes.

Si bien es cierto que reiteradamente se plantea la necesidad de dotar a los centros de recursos, son escasas las referencias a cómo se pueden utilizar las nuevas tecnologías en la gestión de centros escolares y como organizar esta gestión al servicio de la comunidad. Ciertamente, algunos 
centros disponen ya de páginas web, pero se apunta la necesidad de utilizar otras aplicaciones tecnológicas para que no sea el desarrollo tecnológico el que marque la pauta a seguir por no tener definido previamente un modelo que guíe y oriente su utilización al servicio de la comunidad escolar.

\section{Anexo 1. Listado de revistas}

Anales españoles de pediatría

Anuncios

Aula de Innovación Educativa

Aula. Revista de Enseñanza e investigación educativa

Bordón: Revista de Orientación Pedagógica

Ciencia Pediátrica

Ciencia Policial

Comunicar: Revista científica iberoamericana de comunicación y educación

Comunidad Educativa

Control

Cuadernos de Pedagogía

Cultura de los cuidados: Revista de Enfermería y humanidades

Cultura y educación

Documentación social

Economía Industrial

Educación Social

Educación y Biblioteca

Empiria

Enseñanza. Anuario Interuniversitario de Didáctica ISNN 02125374

Familia: revista de Ciencias y Orientación Familiar

Fotogramas

Íber: Didáctica de las Ciencias Sociales, Geografía e Historia

Icono 14

Infancia y sociedad

Investigación en la escuela

Kikiriki: Cooperación educativa

Metas de enfermería 
Organización y Gestión Educativa

Primeras noticias: Comunicación y pedagogía

Psicología Educativa: Revista de los Psicólogos

Red Digital

Revista Complutense de Educación

Revista de estudios de juventud

Revista de psicodidáctica

Revista Española de Pedagogía

Revista iberoamericana de educación

Revista Interuniversitaria de Formación del profesorado

Sociedad y Utopía

Tándem

Telos

Temps d'educació

Trípodos

Viejo Topo

ZER. Revista de Estudios de Comunicación

\section{Bibliografía}

- AGUADED, J.I., 1999, Convivir con la televisión. Familia, educación y recepción televisiva, Piadós, Barcelona.

- GARCÍA GARCÍA, F., 2003, La narrativa hipermedia aplicada a la educación, en Red digital $n^{\circ} 4$.

- GARCÍA MATILLA, A., 2003, Una televisión para la educación: la utopía posible, Gedisa, Barcelona.

- GARCÍA MATILLA, A., 2004, Los niños y los jóvenes frente a las pantallas: Situación de los medios de comunicación y las nuevas tecnologías de la información en España en el ámbito de la infancia y la adolescencia, Ministerio de Trabajo y Asuntos Sociales, Madrid.

- GECA, 2005, Anuario de la televisión, Madrid.

- PABLOS PONS DE, J., 2006, El marco del impacto de las Tecnologías de la Información. Herramientas conceptuales para interpretar la mediación tecnológica educativa, en Telos $n^{\circ}$ 67, Madrid. 
- PABLOS PONS DE, J. y JIMÉNEZ SEGURA, J., 1998, Nuevas tecnologías. Comunicación audiovisual y educación, Cedecs, Barcelona.

- PÉREZ PÉREZ, R., 2000, Nuevas tecnologías de la información y comunicación en la educación. Estado actual y prospectiva, en Telos, n51, Madrid.

- PEREZ TORNERO, J.M., 2000, Educación y televisión en un nuevo proyecto educativo, en Investigación en la escuela n41, Barcelona.

- RODRÍGUEZ ILLERA, J.L., 2004, Las alfabetizaciones digitales, en Bordón: Revista de Orientación Pedagógica, n56, Madrid. 